\title{
Sequential Sensing and Transmission for Real-Time Traffic in Cognitive Networks
}

\author{
Show-Shiow Tzeng ${ }^{1}$ and Ying-Jen Lin ${ }^{2}$ \\ 1 Dept. of Optoelectronics and Communication Engineering, \\ National Kaohsiung Normal Univ., Kaohsiung, Taiwan \\ sstzeng@nknucc.nknu.edu.tw \\ 2 Dept. of Mathematics, National Kaohsiung Normal Univ., Kaohsiung, Taiwan
}

\begin{abstract}
This paper proposes a sequential sensing and transmission algorithm for real-time data in time-slotted multi-band cognitive networks. The proposed algorithm includes the backlog in a queue and the delay constraint of real-time data to select an appropriate band to send data such that more real-time data can be transmitted before being dropped. Simulation results show that the proposed algorithm reduces frames loss ratio and increases effective real-time throughput.
\end{abstract}

Keywords: sequential sensing, real-time traffic, frame loss ratio, multiband, cognitive networks.

\section{Introduction}

Cognitive networks have been introduced to utilize the temporarily idle bands in licensed networks [1. To access an idle spectrum band, the first step of a mobile user in cognitive networks (i.e. cognitive user) is to sense the band and determine whether the band is idle or busy. In a multi-band networks, a cognitive user can use sequential sensing to sense bands one by one. Once one idle band is found, the cognitive user can send out data on the band; otherwise, the user senses the next band. Sequential sensing allows cognitive users to sense two or more bands, which then leads to that cognitive users send out data more quickly in a cost-effective manner 23 .

Previous sequential sensing algorithms implicitly assume that (i) frames are always available from an upper layer at any time and (ii) frames can tolerate long delay. Frames arriving from an upper layer are usually placed into a queue and then be transmitted latter. Due to the first assumption, the number of the frames in a queue is infinite and previous sequential sensing stops to send frames on the band which maximum throughput is the greatest among the sensing bands. However, the number of the frames in a queue is finite and is possibly variable. It is meaningless that a band which can transmit all the frames in a queue is found but we abandon the band and sense the next band due to the reason that the next band may have higher throughput. Therefore, we suggest that sequential sensing may stop on the band which can send out all the frames if the band is found, instead of the band which maximum throughput is the greatest; 
from this viewpoint, the frames can be sent more quickly. Under the second assumption, previous sequential sensing does not consider that real-time frames have the requirement of delay constraint; that is, frames are dropped when the frames suffer a specified delay time. Although sensing more bands may have more opportunity to find a throughput-maximum band, more frames may be also dropped. This paper designs a sequential sensing procedure for real-time traffic in a realistic network environment removing the two assumptions.

\section{The Proposed Sensing and Transmission Algorithm}

The radio environment herein is a licensed network in which radio spectrum is divided into spectrum bands and each band is further divided into time slots. $M$ possible transmission rates are assumed to be available on a band; each rate is denoted by $r_{m}, 1 \leq m \leq M$. The frames, consisting of header and payload, are real-time traffic with the constraint of delay time. The time that a frame stays in a queue is queuing time. The time that a frame is sent out is transmission time. For a frame, if the sum of queuing time and transmission time is greater than the delay requirement, the frame is dropped. Given a set of bands, the amount of real-time data successfully sent in a slot on the bands excludes sensing overhead, header overhead, and the failed data due to miss-detection and dropping. Then, the estimated real-time throughput (ERT) is defined as the ratio of the average amount of real-time data successfully sent in a slot on the bands to a slot time. In the proposed algorithm, a cognitive user starts to sense a time slot on band $b_{i}, i=1$. If sensing result is busy, the user checks whether band $b_{i+1}$ exists. If band $b_{i+1}$ exists, the user senses the same slot on the next band $b_{i+1}$; otherwise, the user waits the beginning of the next slot and then repeats the proposed algorithm. If sensing result is idle, the user calculates (i) the ERT of band $b_{i}$ and (ii) the ERT of the bands $\left\{b_{i+1}, b_{i+2}, \ldots, b_{N}\right\}$; then the user compares the values of the both ERTs. If the ERT of the bands $\left\{b_{i+1}, b_{i+2}, \ldots, b_{N}\right\}$ is higher, the user continues to sense the next band $b_{i+1}$; otherwise, the user sends frames in the residual time of the slot on band $b_{i}$.

In the following, we introduce the notation used in the equations to calculate the ERT, and then the equations are presented. The header length and payload length of a frame are denoted by $L_{h}$ and $L_{d}$ respectively. Let $T_{c}, T_{s}, T_{l}$, and $T_{p}$ respectively denote the time of changing bands, sensing time, slot time, and the probing time of a band. Let $T_{q}^{j}$ denote the queuing time of the $q$-th frame in a queue at the beginning of slot $j$. The probability that a slot is busy (or idle) is denoted by $P_{\text {busy }}\left(\right.$ or $P_{\text {idle }}$ ). The probability that a user correctly detects a busy slot is denoted by $P_{d}$. The probability that a user incorrectly identifies an idle slot as a busy slot is denoted by $P_{f}$. Let $E_{i, k}^{j}$ denote the ERT of slot $j$ on bands $\left\{b_{i}, b_{i+1}, \ldots, b_{N}\right\}$, where $1 \leq i \leq N$ and $k, 0 \leq k \leq i-1$, is the number of probing operations performed before band $b_{i}$. Let $e_{i, k}^{j}(x)$ represent the ERT of slot $j$ on band $b_{i}$, where $x$ is the transmission rate of band $b_{i}$ and $k$ is the number of probing operations performed before band $b_{i}$. We in turn derive $e_{i, k}^{j}(x)$ and $E_{i, k}^{j}$ as follows. When a cognitive user attempts to send frames in a queue in slot $j$ 
on band $b_{i}$, the user experiences $i$ sensing times, $(i-1)$ times of changing bands, and at least one probing time. Since the user may have probed $k$ bands before band $b_{i}$, the $q$-th frame can be sent at a rate $x$ if (i) the frame is sent completely before the end of slot $j$, i.e. $i T_{s}+(i-1) T_{c}+(k+1) T_{p}+q\left(L_{d}+L_{h}\right) / x<T_{l}$ and (ii) the delay constraint $D$ of the frame is satisfied, i.e. $T_{q}^{j}+i T_{s}+(i-1) T_{c}+(k+$ 1) $T_{p}+q\left(L_{d}+L_{h}\right) / x<D$, which can be expressed by an indicator function $\delta_{q}^{j}(x)$. The value of $\delta_{q}^{j}(x)$ is 1 if the frame is sent successfully; otherwise, the value is 0 . The maximum number of frames which can be sent at a rate $x$ in the remaining time of slot $j$ is $\sum_{q \in Q} \delta_{i, k, q}^{j}(x)$, where $Q$ is the set of frames in a queue at the beginning of slot $j$. Then, the ERT, $e_{i, k}^{j}(x)$, is calculated as follows:

$$
e_{i, k}^{j}(x)=\frac{1}{T_{l}} \sum_{q \in Q} \delta_{i, k, q}^{j}(x) L_{d} .
$$

When a user is in slot $j$ on band $b_{i}$, the ERT of bands $\left\{b_{i}, b_{i+1}, \ldots, b_{N}\right\}, E_{i, k}^{j}$, is discussed into three cases: (i) the sensing result of idle band $b_{i}$ is idle, which probability is $P_{\text {idle }}\left(1-P_{f}\right)$, (ii) the sensing result of busy band $b_{i}$ is idle, which probability is $P_{b u s y}\left(1-P_{d}\right)$, and (iii) the sensing result of idle or busy band $b_{i}$ is busy, which probability is $P_{b u s y} P_{d}+P_{i d l e} P_{f}$. In the first case, if the ERT, $e_{i, k}^{j}(x)$, of band $b_{i}$ is greater than or equal to the ERT of bands $\left\{b_{i+1}, b_{i+2}, \ldots, b_{N}\right\}$, the user determines to send frames on band $b_{i}$; i.e., $E_{i, k}^{j}$ is $e_{i, k}^{j}(x)$. Otherwise, the user continues to sense the next band $b_{i+1}$; i.e., $E_{i, k}^{j}$ is $E_{i+1, k+1}^{j}$. Let $\delta_{i, k}^{j}(x)$ be an indicator function which value is 1 if the user sends frames in slot $j$ on band $b_{i}$, i.e. $e_{i, k}^{j}(x) \geq E_{i+1, k+1}^{j}$, and which value is 0 if the user continues to sense. Let $\bar{\delta}_{i, k}^{j}(x)$ denote the complementary of the $\delta_{i, k}^{j}(x)$. The ERT of bands $\left\{b_{i}, b_{i+1}, \ldots, b_{N}\right\}$ in the first case is

$$
\sum_{m=1}^{M} P_{r_{m}}\left[\delta_{i, k}^{j}\left(r_{m}\right) e_{i, k}^{j}\left(r_{m}\right)+\bar{\delta}_{i, k}^{j}\left(r_{m}\right) E_{i+1, k+1}^{j}\right],
$$

where $P_{r_{m}}$ denotes the probability that the transmission rate of band $b_{i}$ is $r_{m}, m=1,2, \ldots, M$. In the second case, when a user probes the rate of band $b_{i}$, the user receives no response due to busy band $b_{i}$. The user continues to sense the next band $b_{i+1}$; then, the ERT, $E_{i, k}$, is $E_{i+1, k+1}^{j}$. In the third case, the user continues sensing from the next band $b_{i+1}$; then, the ERT, $E_{i, k}^{j}$, is $E_{i+1, k}^{j}$. The ERT of bands $\left\{b_{i}, b_{i+1}, \ldots, b_{N}\right\}$ is summarized in recursive Eq. (3) which can be solved by backward induction.

$$
E_{i, k}^{j}=\left\{\begin{array}{l}
P_{i d l e}\left(1-P_{f}\right) \sum_{m=1}^{M} P_{r_{m}}\left[\delta_{i, k}^{j}\left(r_{m}\right) e_{i, k}^{j}\left(r_{m}\right)+\right. \\
\left.\bar{\delta}_{i, k}^{j}\left(r_{m}\right) E_{i+1, k+1}^{j}\right]+P_{b u s y}\left(1-P_{d}\right) E_{i+1, k+1}^{j}+ \\
\left(P_{b u s y} P_{d}+P_{i d l e} P_{f}\right) E_{i+1, k}^{j} \quad 1 \leq i \leq N-1, \\
P_{\text {idle }}\left(1-P_{f}\right) \sum_{m=1}^{M} P_{r_{m}} e_{i, k}^{j}\left(r_{m}\right) \quad i=N .
\end{array}\right.
$$




\section{Simulation Results and Conclusions}

Fig. 1 shows the ERT and frame loss ratio (the ratio of the number of loss frames to the total number of frames) of the proposed algorithm and the two other algorithms; one is the algorithm in [3, which produces maximal effective throughput (MET) without considering delay constraint and the backlog in a queue, and the other is the algorithm, called first-fit herein, which merely sends frames on a band which is first found on a slot and its bandwidth is sufficient to send all the frames in a queue. The unit of the ERT in Fig. 1 is the percentage of the load which can be successfully transmitted. From the figure, we observe that the proposed algorithm reduces frame loss ratio and increases effective real-time throughput because the proposed algorithm includes the backlog and the delay constraint to select an appropriate band to send frames as soon as possible.
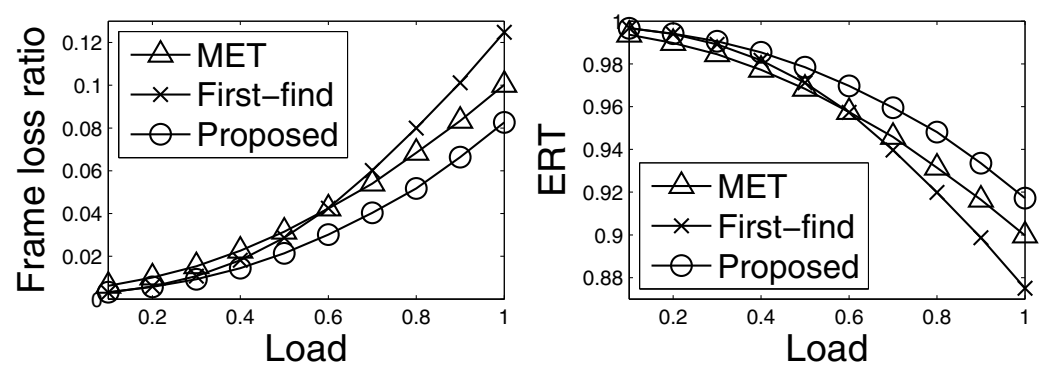

Fig. 1. Frame loss ratio and ERT

Acknowledgement. This research was partially supported by the National Science Council, Taiwan, under grants NSC 101-2221-E-017-011- and NSC 1022221-E-017-006-.

\section{References}

1. Akyildiz, I.F., Lee, W.-Y., Vuran, M.C., Mohanty, S.: A survey on spectrum management in cognitive radio networks. IEEE Comms. Mag. 46(4), 40-48 (2008)

2. Shu, T., Krunz, M.: Throughput-efficient sequential channel sensing and probing in cognitive radio networks under sensing errors. ACM Mobicom 2009, 37-48 (2009)

3. Tzeng, S.-S., Lin, Y.-J.: Cross-layer sequential sensing with effective throughput maximization in time-slotted cognitive networks. Wireless Netw. 19(5), 591-606 (2013) 